\title{
Interosseous Artery
}

National Cancer Institute

\section{Source}

National Cancer Institute. Interosseous Artery. NCI Thesaurus. Code C32864.

Either of three arteries including the common interosseous artery which arises from the ulnar artery and bifurcates into the anterior and posterior interosseous arteries. 